\title{
OSCILLATION AND NONOSCILLATION CRITERIA FOR DELAY DIFFERENTIAL EQUATIONS
}

\author{
Á. ELBERT AND I. P. STAVROULAKIS
}

(Communicated by Hal L. Smith)

\begin{abstract}
Oscillation and nonoscillation criteria for the first-order delay differential equation
\end{abstract}

$$
x^{\prime}(t)+p(t) x(\tau(t))=0, \quad t \geq t_{0}, \tau(t)<t,
$$

are established in the case where

$$
\int_{\tau(t)}^{t} p(s) d s \geq \frac{1}{e} \text { and } \quad \lim _{t \rightarrow \infty} \int_{\tau(t)}^{t} p(s) d s=\frac{1}{e}
$$

\section{INTRODUCTION}

The qualitative properties of the solutions of the delay differential equation

$$
x^{\prime}(t)+p(t) x(\tau(t))=0, \quad . t \geq t_{0},
$$

where $\tau(t)<t$, have been the subject of many investigations. The first systematic study was made by Myshkis [6]. Among others he has shown [5] that all solutions of (1) oscillate if

$$
p(t) \geq 0, \quad \quad \quad \limsup [t-\tau(t)]<\infty, \quad \quad \liminf _{t \rightarrow \infty}[t-\tau(t)] \cdot \liminf _{t \rightarrow \infty} p(t)>\frac{1}{e} .
$$

Later these conditions were improved, by Ladas [4] and Koplatadze and Chanturija [3], to

$$
\liminf _{t \rightarrow \infty} \int_{\tau(t)}^{t} p(s) d s>\frac{1}{e}
$$

Concerning the constant $\frac{1}{e}$ in (2) we mention that if the inequality

$$
\int_{\tau(t)}^{t} p(s) d s \leq \frac{1}{e}
$$

holds, then, according to a result in [3], (1) has a nonoscillatory solution. To the best of our knowledge there is no result in the case when we have

$$
\int_{\tau(t)}^{t} p(s) d s \geq \frac{1}{e} \quad \text { and } \quad \lim _{t \rightarrow \infty} \int_{\tau(t)}^{t} p(s) d s=\frac{1}{e} .
$$

Received by the editors August 26, 1993.

1991 Mathematics Subject Classification. Primary 34K15; Secondary 34C10.

Key words and phrases. Oscillation, nonoscillation, delay differential equations. 
In connection with the delay function $\tau(t)$ in (1) we suppose that $\tau(t)$ is strictly increasing on $\left[t_{0}, \infty\right), \lim _{t \rightarrow \infty} \tau(t)=\infty$, and its inverse is $\tau_{-1}(t)\left(\tau_{-1}(t)>t\right)$. Let $\tau_{-k}(t)$ be defined on $\left[t_{0}, \infty\right)$ by

$$
\tau_{-k-1}(t)=\tau_{-1}\left(\tau_{-k}(t)\right) \text { for } k=1,2, \ldots,
$$

and let

$$
t_{k}=\tau_{-k}\left(t_{0}\right), \quad k=1,2, \ldots .
$$

Clearly $t_{k} \rightarrow \infty$ as $k \rightarrow \infty$.

The coefficient $p(t)$ is assumed to be a piecewise continuous function and satisfies the relation

$$
\int_{\tau(t)}^{t} p(s) d s \geq \frac{1}{e} .
$$

Let $\varphi(t)$ be a continuous function on $\left[\tau\left(t_{0}\right), t_{0}\right]$. A function $x(t)$ is a solution of (1), associated with the initial function $\varphi(t)$, if $x(t)=\varphi(t)$ on $\left[\tau\left(t_{0}\right), t_{0}\right], x(t)$ is continuous on $\left[\tau\left(t_{0}\right), \infty\right)$, is differentiable almost everywhere on $\left(t_{0}, \infty\right)$, and satisfies (1).

As is customary, a solution is called oscillatory if it has arbitrarily large zeros. Otherwise it is called nonoscillatory.

Among the functions $p(t)$ we define a set $\mathscr{A}_{\lambda}$ for $0<\lambda \leq 1$ as follows.

Definition. The piece-wise continuous function $p(t):\left[t_{0}, \infty\right] \rightarrow[0, \infty]$ belongs to $\mathscr{A}_{\lambda}$ if

$$
\int_{\tau(t)}^{t} p(s) d s \geq \frac{1}{e}, \quad t \geq t_{1}
$$

$$
\int_{\tau(t)}^{t} p(s) d s \geq \frac{1}{e}+\lambda_{k}\left(\int_{t_{k}}^{t_{k+1}} p(s) d s-\frac{1}{e}\right) \quad \text { for } t_{k}<t \leq t_{k+1}, k=1,2, \ldots,
$$

for some $\lambda_{k} \geq 0$, and

$$
\liminf _{k \rightarrow \infty} \lambda_{k}=\lambda>0
$$

We remark that if $\int_{\tau(t)}^{t} p(s) d s$ is a nonincreasing function and $\int_{\tau(t)}^{t} p(s) d s>$ $\frac{1}{e}$, then $p(t) \in \mathscr{A}_{1}$, because we may have $\lambda_{k}=1$ in (5). However, the monotonicity is not a necessary condition; e.g., in the case $\tau(t)=t-1$ the function

$$
p(t)=\frac{1}{e}+\left(K \sin ^{2} \pi t / t^{\alpha}\right), \quad K>0 \text { and } 0 \leq \alpha \leq 2,
$$

belongs to $\mathscr{A}_{1}$ because $\int_{t-1}^{t}\left(\sin ^{2} \pi s / s^{\alpha}\right) d s$ is a nonincreasing function.

Our main results are

Theorem 1. Assume that the function $p(t)$ in (1) belongs to $\mathscr{A}_{\lambda}$ for some $\lambda \in$ $(0,1]$ and

$$
\sum_{i=1}^{\infty}\left(\int_{t_{i-1}}^{t_{i}} p(s) d s-\frac{1}{e}\right)=+\infty .
$$

Then every solution of (1) oscillates. 
In the next theorem we consider the case where the sum in (7) is convergent.

Theorem 2. Assume that $p(t) \in \mathscr{A}_{\lambda}$, for some $0<\lambda \leq 1$ and either

$$
\lambda \underset{k \rightarrow \infty}{\limsup } k \sum_{i=k}^{\infty}\left(\int_{t_{i-1}}^{t_{i}} p(s) d s-\frac{1}{e}\right)>\frac{2}{e}
$$

or

$$
\lambda \liminf _{k \rightarrow \infty} k \sum_{i=k}^{\infty}\left(\int_{t_{i-1}}^{t_{i}} p(s) d s-\frac{1}{e}\right)>\frac{1}{2 e} .
$$

Then every solution of (1) oscillates.

Note. If the function $\int_{\tau(t)}^{t} p(s) d s$ is monotone, then the value of $\lambda$ in conditions (8) and (9) of Theorem 2 is equal to one.

In the following theorem we give a criterion for nonoscillation.

Theorem 3. Let $\tau(t)=t-1, p(t)=\frac{1}{e}+a(t)$, and $t_{0}=1$ in (1); i.e., it has the form

$$
x^{\prime}(t)+\left[\frac{1}{e}+a(t)\right] x(t-1)=0, \quad t \geq 1
$$

Assume that

$$
a(t) \leq 1 / 8 e t^{2}
$$

Then (1)' has a soluion $x(t) \geq \sqrt{t} e^{-t}$.

The proofs of the above theorems and also some lemmas which will be used in these proofs will be given in the next section.

\section{LEMMAS AND PROOFS}

The first two lemmas have origin in [3] (see also [2]).

Lemma 1. Assume that $x(t)$ is a positive solution of (1) on $\left[t_{k-2}, t_{k+1}\right]$ for some $k \geq 2$. Let $N$ be defined by

$$
N=\min _{t_{k} \leq t \leq t_{k+1}} \frac{x(\tau(t))}{x(t)} .
$$

Then $N<(2 e)^{2}$.

Proof. Let $L$ be the integral

$$
L=\int_{t_{k}}^{t_{k+1}} p(s) d s \geq \frac{1}{e} .
$$

By Lemma 3 in [2], we obtain $N<((1+\sqrt{1-L}) / L)^{2}$. Since the right-hand side is a decreasing function of $L$, we get

$$
N<((1+\sqrt{1-(1 / e)}) / L)^{2}<(2 e)^{2} .
$$

Lemma 2. Assume that $x(t)$ is a positive solution of (1) on $\left[t_{k-3}, t_{k+1}\right]$ for some $k \geq 3$ and $p(t) \in \mathscr{A}_{\lambda}$. Let $M, N$ be defined by

$$
M=\min _{t_{k-1} \leq t \leq t_{k}} \frac{x(\tau(t))}{x(t)}, \quad N=\min _{t_{k} \leq t \leq t_{k-1}} \frac{x(\tau(t))}{x(t)} .
$$


Then

$$
M>1 \text { and } N \geq \exp \left(M\left[\frac{1}{e}+\lambda_{k}\left(\int_{t_{k}}^{t_{k+1}} p(s) d s-\frac{1}{e}\right)\right]\right) \geq M .
$$

Proof. Following the lines of the proof of Lemma 1 in [2], we have $\min \{M, N\}$ $=M$, and by (5) for $t_{k} \leq t \leq t_{k+1}$

$$
\frac{x(\tau(t))}{x(t)} \geq \exp \left(M \int_{\tau(t)}^{t} p(s) d s\right) \geq \exp \left(M\left[\frac{1}{e}+\lambda_{k}\left(\int_{t_{k}}^{t_{k+1}} p(s) d s-\frac{1}{e}\right)\right]\right),
$$

which implies the inequality concerning $N$. On the other hand the solution $x(t)$ is a strictly decreasing function on $\left[t_{k-2}, t_{k+1}\right]$. Hence $x(\tau(t)) / x(t)>1$ on $\left[t_{k-1}, t_{k}\right]$, and therefore $M>1$. The proof of the lemma is complete.

The next lemma deals with some properties of the following sequence.

Let the sequence $\left\{r_{i}\right\}_{i=0}^{\infty}$ be defined by the recurrence relation

$$
r_{0}=1, \quad r_{i+1}=e^{r_{i} / e} \quad \text { for } i=0,1,2, \ldots .
$$

Lemma 3. For the sequence $\left\{r_{i}\right\}_{i=0}^{\infty}$ in (10) the following relations hold:
(a) $r_{i}<r_{i+1}$;
(b) $r_{i}<e$
(c) $\lim _{i \rightarrow \infty} r_{i}=e$;
(d) $r_{i}>e-2 e /(i+2)$.

Proof. The first two relations can be proved by induction. As a consequence of (a) and (b) the $\lim _{i \rightarrow \infty} r_{i}=r$ exists and it is finite. Then by (10) we have

$$
r=e^{r / e} \text {. }
$$

It is easy to check that

$$
e^{x / e}>x \text { for } x \neq e .
$$

This inequality implies that the limit $r$ equals $e$.

Now we give the proof of (d). For $i=0$ and $i=1$ it is immediate. For $i \geq 1$ the proof goes by induction, so we have

$$
r_{i+1}=e^{r_{i} / e}>e^{1-2 /(i+2)},
$$

and it is sufficient to show

or

$$
e^{1-2 /(i+2)}>e-\frac{2 e}{i+3}
$$

$$
f(x)=e^{-2 / x}+\frac{2}{x+1}>1 \text { for } x=i+2 .
$$

Since

$$
f^{\prime}(x)=\frac{2}{x^{2}}\left(e^{-1 / x}+\frac{x}{x+1}\right)\left(e^{-1 / x}-\frac{x}{x+1}\right)
$$

and

$$
e^{1 / x}>1+\frac{1}{x}=\frac{x+1}{x}
$$

we have $f^{\prime}(x)<0$ and $f(x)>\lim _{x \rightarrow \infty} f(x)=1$, which was to be shown.

The proof of the lemma is complete. 
Proof of Theorem 1. Suppose the contrary. Then we may assume, without loss of generality, that there exists a solution $x(t)$ such that $x(t)>0$ for $t \geq t_{k-3}$ for some $k \geq 3$. Let the sequence $\left\{N_{i}\right\}_{i=0}^{\infty}$ be defined by

$$
N_{i}=\min _{t_{k+i-1} \leq t \leq t_{k+i}} \frac{x(\tau(t))}{x(t)} .
$$

By Lemma 2 we have $N_{0}>1$ and

$$
N_{i+1} \geq \exp \left(\frac{N_{i}}{e}\right) \exp \left(N_{i} \lambda_{k+i}\left(\int_{t_{k+i}}^{t_{k+i+1}} p(s) d s-\frac{1}{e}\right)\right) \geq N_{i} ;
$$

therefore the sequence $\left\{N_{i}\right\}_{i=0}^{\infty}$ is nondecreasing. On the other hand, by Lemma 1 , it is bounded. Consequently the sequence converges. Let

$$
\lim _{i \rightarrow \infty} N_{i}=N \text {. }
$$

Then (13) implies

$$
N \geq \exp (N / e) .
$$

Hence by (11) we have $N=e$ and

$$
1<N_{0}<N_{1}<\cdots<e \text {. }
$$

From (13), in view of (11), we obtain

$$
N_{i+1} \geq N_{i}\left(1+N_{i} \lambda_{k+i}\left(\int_{t_{k+i}}^{t_{k+i+1}} p(s) d s-\frac{1}{e}\right)\right) .
$$

Thus

$$
\dot{N}_{i+1}-N_{i}>N_{i}^{2} \lambda_{k+i}\left(\int_{t_{k+i}}^{t_{k+i+1}} p(s) d s-\frac{1}{e}\right) .
$$

From the definition of $\mathscr{A}_{\lambda}$ we know that $\lambda=\liminf _{k \rightarrow \infty} \lambda_{k}>0$, so for any sufficiently small $\varepsilon>0$ there exists a value $\kappa_{\varepsilon}$ such that $\lambda_{k+i}>\lambda-\varepsilon$ for $k+i>\kappa_{\varepsilon}$. Thus, for such $i$ 's from (15) and (14), we have

$$
\begin{aligned}
N_{i+1}-N_{i} & >N_{i}^{2}(\lambda-\varepsilon)\left(\int_{t_{k+i}}^{t_{k+i+1}} p(s) d s-\frac{1}{e}\right), \\
N_{i+2}-N_{i+1} & >N_{i+1}^{2}(\lambda-\varepsilon)\left(\int_{t_{k+i+1}}^{t_{k+i+2}} p(s) d s-\frac{1}{e}\right) \\
& \geq N_{i}^{2}(\lambda-\varepsilon)\left(\int_{t_{k+i+1}}^{t_{k+i+2}} p(s) d s-\frac{1}{e}\right) .
\end{aligned}
$$

Summing up the inequalities above, we obtain

$$
e-N_{i}>N_{i}^{2}(\lambda-\varepsilon) \sum_{j=i}^{\infty}\left(\int_{t_{k+j}}^{t_{k+j+1}} p(s) d s-\frac{1}{e}\right) \quad \text { for } k+i \geq \kappa_{\varepsilon} .
$$

The last inequality contradicts assumption (7). The proof is complete. 
Proof of Theorem 2. Suppose the contrary. Then, as in the proof of Theorem 1, we have the sequence $\left\{N_{i}\right\}_{i=0}^{\infty}$ such that inequalities (13)-(16) hold. In particular, from (13) we have

$$
N_{i+1} \geq \exp \left(N_{i} / e\right)
$$

Comparing the last inequality with (10), we obtain by induction

$$
N_{0}>r_{0}=1, \quad N_{i}>r_{i} \quad \text { for } i=1,2, \ldots .
$$

Then by Lemma $3(\mathrm{~d})$ we have

$$
e-N_{i}<e-r_{i}<2 e /(i+2) .
$$

Multiplying (16) by $k+i$ we obtain

$$
(k+i) \frac{2 e}{i+2}>N_{i}^{2}(\lambda-\varepsilon)(k+i) \sum_{j=k+i}^{\infty}\left(\int_{t_{j}}^{t_{j+1}} p(s) d s-\frac{1}{e}\right) \quad \text { for } k+i \geq \kappa_{\varepsilon} .
$$

Taking the limit as $i \rightarrow \infty$ we get

$$
2 e \geq e^{2} \lambda \limsup _{k \rightarrow \infty} k \sum_{j=k}^{\infty}\left(\int_{t_{j}}^{t_{j+1}} p(s) d s-\frac{1}{e}\right)
$$

which contradicts $(8)$.

Now let $A$ be defined by

$$
A=\liminf _{k \rightarrow \infty} k \sum_{j=k}^{\infty}\left(\int_{t_{j}}^{t_{j+1}} p(s) d s-\frac{1}{e}\right) .
$$

If $A=\infty$, then, by (8), every solution oscillates. Therefore we consider the case $0<A<\infty$. So for any sufficiently small $\varepsilon>0$ there exists a value $\hat{\kappa}_{\varepsilon}$ such that for $\hat{\lambda}=\lambda-\varepsilon>0$ and $\hat{A}=A-\varepsilon>0$

$$
\lambda_{k}>\hat{\lambda} \text { and } \sum_{j=k}^{\infty}\left(\int_{t_{j}}^{t_{j+1}} p(s) d s-\frac{1}{e}\right)>\frac{\hat{A}}{k} \quad \text { for } k \geq \hat{\kappa}_{\varepsilon} .
$$

If we use the inequality

$$
\exp \frac{x}{e}>x+\frac{1}{2} \exp \left(\frac{\xi}{e}\right)\left(1-\frac{x}{e}\right)^{2} \text { for } \xi<x<e
$$

in (13) we obtain for $N_{i}>\xi$ and $k+i>\hat{\kappa}_{\varepsilon}$

$$
\begin{aligned}
N_{i+1} & \geq \exp \left(\frac{N_{i}}{e}\right) \exp \left(N_{i} \hat{\lambda}\left(\int_{t_{k+i}}^{t_{k+i+1}} p(s) d s-\frac{1}{e}\right)\right) \\
& >\left[N_{i}+\frac{1}{2} \exp \left(\frac{\xi}{e}\right)\left(1-\frac{N_{i}}{e}\right)^{2}\right]\left(1+N_{i} \hat{\lambda}\left(\int_{t_{k+i}}^{t_{k+i+1}} p(s) d s-\frac{1}{e}\right)\right) .
\end{aligned}
$$

Consequently

$$
N_{i+1}-N_{i}>\frac{1}{2} \exp \left(\frac{\xi}{e}\right)\left(1-\frac{N_{i}}{e}\right)^{2}+\xi^{2} \hat{\lambda}\left(\int_{t_{k+i}}^{t_{k+i+1}} p(s) d s-\frac{1}{e}\right)
$$


and summing up,

$$
e-N_{i}>\frac{1}{2} \exp \left(\frac{\xi}{e}\right) \sum_{j=i}^{\infty}\left(1-\frac{N_{i}}{e}\right)^{2}+\xi^{2} \hat{\lambda} \sum_{j=k+i}^{\infty}\left(\int_{t_{j}}^{t_{j+1}} p(s) d s-\frac{1}{e}\right)
$$

or

$$
e-N_{i}>\frac{1}{2} \exp \left(\frac{\xi}{e}\right) \sum_{j=i}^{\infty}\left(1-\frac{N_{i}}{e}\right)^{2}+\frac{\xi^{2} \hat{\lambda} \hat{A}}{k+i}
$$

In particular the last inequality yields

$$
e-N_{i}>U_{0} /(k+i), \quad U_{0}=\xi^{2} \hat{\lambda} \hat{A} .
$$

By iteration we can improve this inequality to

$$
e-N_{i}>\frac{U_{n}}{k+i}, \quad n=0,1,2, \ldots
$$

Namely by (19) we have

$$
\begin{aligned}
e-N_{i} & >\frac{1}{2} \exp \left(\frac{\xi}{e}\right) \sum_{j=i}^{\infty}\left(\frac{U_{n}}{e(k+j)}\right)^{2}+\frac{\xi^{2} \hat{\lambda} \hat{A}}{k+i} \\
& >\frac{U_{n}^{2}}{2 e^{2}} \exp \left(\frac{\xi}{e}\right) \frac{1}{k+i}+\frac{\xi^{2} \hat{\lambda} \hat{A}}{k+i}=\frac{U_{n+1}}{k+i},
\end{aligned}
$$

where

$$
U_{n+1}=\frac{U_{n}^{2}}{2 e^{2}} \exp \left(\frac{\xi}{e}\right)+\xi^{2} \hat{\lambda} \hat{A}, \quad n=0,1,2, \ldots
$$

From this it is clear that the sequence $\left\{U_{n}\right\}_{n=0}^{\infty}$ is increasing. Moreover, comparing inequalities (17) and (20) we see that $U_{n} \leq 2 e$. Therefore the sequence has a limit, say $U$, which satisfies the equation

$$
U=\frac{U^{2}}{2 e^{2}} \exp \left(\frac{\xi}{e}\right)+\xi^{2} \hat{\lambda} \hat{A}
$$

This is a quadratic equation with real roots and therefore the descriminant is not negative; i.e.,

$$
1-2 e^{\xi / e-2} \xi^{2} \hat{\lambda} \hat{A} \geq 0 .
$$

Let $\varepsilon \rightarrow 0$ and $\xi \rightarrow e$. Then the last inequality becomes

$$
1-2 e \lambda A \geq 0
$$

which contradicts (9).

The proof of the theorem is complete.

Proof of Theorem 3. The proof is based on known comparison theorems (see Myshkis [6] or Elbert [1]). Let the functions $A(t), B(t), C(t)$ be defined as

$$
\begin{aligned}
& A(t)=\frac{1}{e}+a(t), \\
& B(t)=\frac{1}{e}+\frac{1}{8 e t^{2}}, \\
& C(t)=\frac{1}{e} \frac{1-\frac{1}{2 t}}{\sqrt{1-\frac{1}{t}}}, \quad t>1 .
\end{aligned}
$$


By the assumption we have $A(t) \leq B(t)$. We are going to show that the inequality $B(t)<C(t)$ also holds. Namely, for $\theta=\frac{1}{2 t} \in\left(0, \frac{1}{2}\right)$, we have

$$
C(t)-B(t)=\frac{\theta^{3}\left(\frac{1}{2} \theta^{2}-\frac{1}{4} \theta+2\right)}{e \sqrt{1-2 \theta}\left[1-\theta+\left(1+\frac{1}{2} \theta^{2}\right) \sqrt{1-2 \theta}\right.}>0 .
$$

Now we will compare the differential equations

$$
\begin{aligned}
& x^{\prime}(t)+A(t) x(t-1)=0, \\
& z^{\prime}(t)+B(t) z(t-1)=0, \\
& u^{\prime}(t)+C(t) u(t-1)=0 .
\end{aligned}
$$

Let us observe that the function $u(t)=\sqrt{t} e^{-t}$ is a solution of the last differential equation. Let the initial function $\varphi(t)$ be the function $\sqrt{t} e^{-t}$ on $[0,1]$, and let $x(t)$ and $z(t)$ be the solutions of the first and the second differential equations respectively, associated with this initial function $\varphi(t)$. Then by the comparison theorems mentioned above we have

$$
x(t) \geq z(t)>u(t)=\sqrt{t} e^{-t} \text { for } t>1 .
$$

which was to be shown.

Remark 1. For $(1)^{\prime}$ we have $t_{k}=k+1$ and

$$
\limsup _{k \rightarrow \infty} k \sum_{i=k}^{\infty}\left(\int_{t_{i-1}}^{t_{i}} p(s) d s-\frac{1}{e}\right)=\limsup _{k \rightarrow \infty} k \int_{k}^{\infty} a(t) d t \leq \frac{1}{8 e} .
$$

Now the question arises naturally whether or not the bounds in conditions (8) and (9) of Theorem 2 can be replaced by smaller ones.

Remark 2. It is to be emphasized that in Theorem 3 we require neither

$$
p(t) \geq 0 \text { nor } \int_{\tau(t)}^{t} p(s) d s \geq \frac{1}{e} .
$$

Remark 3. Applying Theorems 1, 2 we see that, under (6), (1) oscillates for any $K>0$ if $0 \leq \alpha<2$ and for $K>\frac{1}{e}$ if $\alpha=2$. On the other hand it has a nonoscillatory solution for $K<\frac{1}{8 e}$ if $\alpha=2$.

\section{REFERENCES}

1. Á. Elbert, Comparison theorem for first order nonlinear differential equations with delay, Studia Sci. Math. Hungar. 11 (1976), 259-267.

2. Á Elbert and I. P. Stavroulakis, Oscillations of first order differential equations with deviating arguments, World Sci. Ser. Appl. Anal., vol. 1, World Sci. Publishing, Teaneck, NJ, 1992, pp. 163-178.

3. R. G. Koplatadze and T. A. Chanturija, On the oscillatory and monotonic solutions of first order differential equations with deviating arguments, Differentsial'nye Uravneniya 18 (1982), 1463-1465. (Russian)

4. G. Ladas, Sharp conditions for oscillations caused by delays, Appl. Anal. 9 (1979), 93-98.

5. A. D. Myshkis, Linear homogeneous differential equations of first order with deviating arguments, Uspekhi Mat. Nauk 5 (1950), 160-162. (Russian)

6. L Linear differential equations with retarded argument, 2nd edition, "Nauka", Moscow, 1972. (Russian)

Mathematical Institute, Hungarian Academy of Sciences, Budapest, 1364 Hungary

Department of Mathematics, University of Ioannina, 45110 Ioannina, Greece 